\title{
Thin-Film Gold Electrodes Produced by Magnetron Sputtering. Voltammetric Characteristics and Application in Batch Injection Analysis with Amperometric Detection
}

\author{
Ana Maria Oliveira Brett, ${ }^{*+}$ Frank-Michael Matysik, ${ }^{+}$and M. Teresa Vieira ${ }^{++}$ \\ + Departamento de Química, Faculdade de Ciências e Tecnologia, Universidade de Coimbra, P-3000 Coimbra, Portugal \\ ${ }^{++}$Departamento de Engenharia Mecânica, Faculdade de Ciências e Tecnologia, Universidade de Coimbra, P-3000 Coimbra, Portugal
}

Received: June 27, 1996

Final version: August 12, 1996

\begin{abstract}
Thin-film gold electrodes were produced by radio-frequency sputtering using a polyvinyl chloride foil as substrate. The voltammetric behavior was characterized in various electrolyte solutions. In alkaline and neutral aqueous solutions reliable voltammetric measurements can be performed while in acidic media some nondefined phenomena occur. A novel type of electrode for batch injection analysis (BIA) is presented which is produced from circular pieces of the thin-film material. This electrode can be used for BIA in conventional voltammetric cells. Pulsed amperometric detection is demonstrated to be viable with thin-film gold electrodes and shows analytical advantages over constant-potential detection in the BIA of hydrogen peroxide.
\end{abstract}

Keywords: Batch injection analysis, Thin-film gold electrode, Magnetron sputtering, Multiple pulse detection

\section{Introduction}

During recent years much progress has been made in developing new fabrication techniques for electrode materials. For the deposition of noble metals on various substrates, a variety of methods can be used. For example, gold films can be prepared by thermal evaporation [1], sputtering [2] chemical vapor deposition [3], the ionized cluster beam technique [4] or high speed selective jet electrodeposition [5]. However, the electrical and electrochemical parameters can be very different and the applicability of some of these gold-plated substrates for electrochemical purposes is questionable or depends on the parameters chosen in the film production. In any case the suitability of the proposed material for electrochemical measurements has to be evaluated. From the work referred to above, only reference [1] involves electrochemical characterization of the gold film material.

Screen-printing [6] and ink-printing [7] have been proposed for the fabrication of disposable electrodes. The main aims of these electrodes are in the fabrication of inexpensive enzyme sensors [8] or as simple devices for stripping analysis [9]. The preparation of noble metal films on thin plastic sheets is attractive because electrodes of any size or shape can easily be cut and used for particular electrochemical purposes.

In this article we report the voltammetric behavior of thin-film gold electrodes prepared by cathodic sputtering using a plastic sheet as substrate. We show that using this material can lead to a new type of electrode for batch injection analysis (BIA) [10]. It will be demonstrated that pulsed amperometric detection [11] is also possible with the thin-film gold electrodes and can be utilized for the detection of hydrogen peroxide.

\section{Experimental}

\subsection{Chemicals and Materials}

All solutions were prepared from commercial analytical grade chemicals excepting potassium hexacyanoosmate(II) which was obtained from Dr. R. Billing (University of Leipzig, Germany). Millipore Milli-Q nanopure water was used throughout for the preparation and dilution of solutions. Hydrogen peroxide solutions were prepared freshly prior to the experiments by diluting a $35 \% \mathrm{w} . / \mathrm{w}$. solution $\left(d=1.135 \mathrm{~g} \mathrm{~cm}^{-3}\right)$.

Silver epoxy (RS Components, England) was used to join a copper wire to the thin-film gold electrodes. Nonconductive epoxy (Conrad Electronics, Germany) served for encapsulation of the conductive joint.

\subsection{Preparation of Thin-Film Gold Electrodes}

The gold thin film was deposited by magnetron sputtering using an Edwards Model E306A from a pure gold target with ionized argon onto a polyvinyl chloride (PVC) substrate sheet of thickness $0.08 \mathrm{~mm}$. The radio frequency (r.f.) magnetron sputtering enables deposition of thin films of metals at controlled rates onto nonconducting substrates without thermal destruction. In this case the deposition temperature is inferior to the glass transition temperature of PVC.

In order to obtain the optimum deposition conditions for the adhesion of the gold film to the substrate several parameters were varied. The optimized conditions found were: deposition power $P / A=11 \mathrm{~W} \mathrm{~cm}^{-2}$, deposition time $2 \mathrm{~min}$, initial pressure (evacuation) $10^{-3} \mathrm{~Pa}$, ultimate pressure (with injected argon) $0.5 \mathrm{~Pa}$. Only those thin-film gold electrodes with optimized adhesion properties were studied with respect to their electrochemical behavior.

After removal from the deposition chamber the film was bombarded by a very thin jet of fine sand in order to create a network of gold on the PVC surface. In this way it was possible to form a gold film with very small bare areas. The electrical conductivity of the gold film was not disrupted, it was suitable for enzyme covalent bonding, and also had light pathways enabling this electrode to be used for spectroelectrochemical purposes. Film morphology was examined with a Jeol T330 scanning electron microscope and showed a uniform compact featureless gold film of thickness less than $1 \mu \mathrm{m}$ with good adhesion.

Circular pieces $(d=8 \mathrm{~mm})$ were cut from the gold plastic sheets and electrodes were constructed as illustrated in Figure 1.

\subsection{Voltammetric and Batch Injection Equipment}

Experiments were performed in a standard 3-electrode voltammetric cell consisting of a platinum coil counter electrode, a 


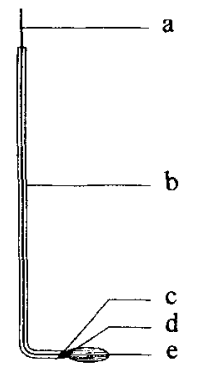

Fig. 1. Construction of circular shaped thin-film gold electrodes used for batch injection analysis: a) Electrical contact of the electrode lead; b) glass tube ( $2 \mathrm{~mm}$ o.d.); c) nonconductive epoxy encapsulation; d) silver epoxy joint; e) electrode surface.

$\mathrm{Ag} / \mathrm{AgCl} / 3 \mathrm{M} \mathrm{KCl}$ reference electrode and a thin-film gold working electrode.

In some experiments, a small spherical gold electrode was used as working electrode. This electrode was prepared by soldering a gold wire of $2 \mathrm{~cm}$ length and $100 \mu \mathrm{m}$ diameter to a copper lead and melting the end of the gold wire in a micro torch to form a gold sphere of about $0.6 \mathrm{~mm}$ diameter. The gold sphere was fixed at the end of a glass tube with epoxy exposing about $3 / 4$ of its surface to the solution.

All voltammetric measurements were done with a computer controlled potentiostat (PGSTAT 10, Eco Chemie, Utrecht, Netherlands). A programmable motorized electronic micropipette (EDP-Plus EP-100, Rainin Instrument Co. Inc.) was used for performing injections. The pipette tip was positioned $2 \pm 0.5 \mathrm{~mm}$ above the center of the thin-film electrode.

\section{Results and Discussion}

\subsection{Voltammetric Behavior of Thin-Film Gold Electrodes in Different Electrolyte Solutions}

The voltammetric properties of the thin-film gold electrodes were characterized by performing measurements in various aqueous solutions, namely $0.1 \mathrm{M}$ sodium hydroxide, $0.5 \mathrm{M}$ potassium chloride, $0.05 \mathrm{M}$ phosphate buffer ( $\mathrm{pH} 7.05$ ), $0.05 \mathrm{M}$ acetate buffer (pH 4.6) and $1 \mathrm{M}$ perchloric acid. The results were compared with those obtained using the small spherical gold electrode. This electrode was found to exhibit typical behavior of polycrystalline gold.

In alkaline solution $(0.1 \mathrm{M} \mathrm{NaOH})$ the thin-film gold electrodes could be employed within the whole potential region between hydrogen evolution $(-1.2 \mathrm{~V})$ and oxygen evolution $(0.75 \mathrm{~V})$. In potassium chloride solution well-defined voltammograms could be recorded using the thin-film electrodes within a potential window between $0 \mathrm{~V}$ and $0.8 \mathrm{~V}$. However, applying negative potentials resulted in an additional anodic wave starting at $0.1 \mathrm{~V}$. Also, potentials more positive than $0.8 \mathrm{~V}$ should be avoided because irreversible damage to the thin gold film can occur. Figure 2 shows a well-defined cyclic voltammogram obtained with a thin-film gold electrode in a solution containing two cyanometalates within the useful potential range for voltammetric determinations in potassium chloride electrolyte. In addition, square-wave and differential pulse measurements were performed using these model analytes. Reproducible results and stable electrode response were obtained throughout.

Measurements in phosphate buffer solution, illustrated in Figure 3 , show similar behavior for the thin-film gold electrode and the spherical gold electrode. There are several small differences. At the thin-film gold electrode a small anodic wave can be seen preceding

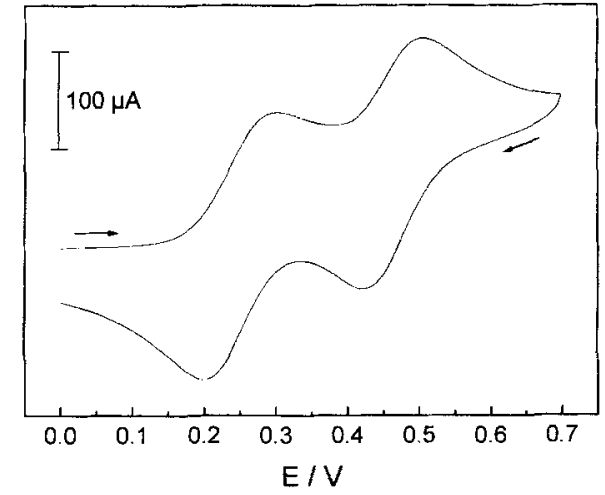

Fig. 2. Cyclic voltammogram obtained with a thin-film gold electrode in a $5 \mathrm{mM} \mathrm{K}_{4}\left[\mathrm{Fe}(\mathrm{CN})_{6}\right], 5 \mathrm{mM} \mathrm{K} \mathrm{K}_{4}\left[\mathrm{Os}(\mathrm{CN})_{6}\right]$ and $0.5 \mathrm{M} \mathrm{KCl}$ solution. Scan rate $100 \mathrm{mV} \mathrm{s}^{-1}$.

the more massive oxide layer formation which starts at more positive potentials than at the spherical gold electrode. The peak potentials for the reduction of the oxide layer are the same, but the reduction peak obtained with the thin-film gold electrode shows a shoulder. Regarding the effects arising from the application of negative potentials, the same behavior was observed as described for potassium chloride solutions. In contrast to potassium chloride solutions, quite positive potentials, even in the region of oxygen evolution, can be used in phosphate buffer solutions without the danger of damaging the electrode.

The voltammetric behavior of the thin-film gold electrodes in acidic solution (perchloric acid and acetate buffer) was not so good with respect to repeatability. The reason for the nondefined behavior could be the limited stability of the material used for electrode construction including the encapsulation components. However, this was not studied in greater detail because the main applications of the thin-film gold electrode are intended to be concerned with biologically important systems in neutral media.

\subsection{Hydrogen Peroxide Detection}

The current-potential characteristics of hydrogen peroxide oxidation at thin-film gold electrodes were studied using a batch injection arrangement. Figure $4 \mathrm{~B}$ shows a cyclic voltammogram
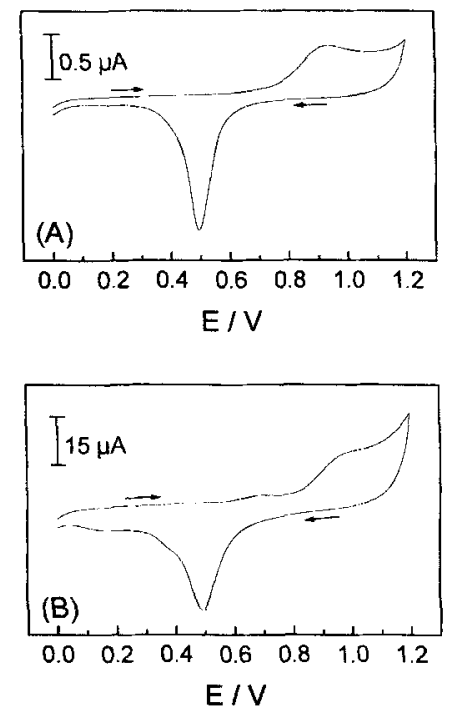

Fig. 3. Cyclic voltammograms in phosphate buffer solution $(50 \mathrm{mM}$, $\mathrm{pH}$ 7.05). Scan rate $50 \mathrm{mV} \mathrm{s}^{-1}$. A) Spherical gold electrode, B) Thin-film gold electrode. 

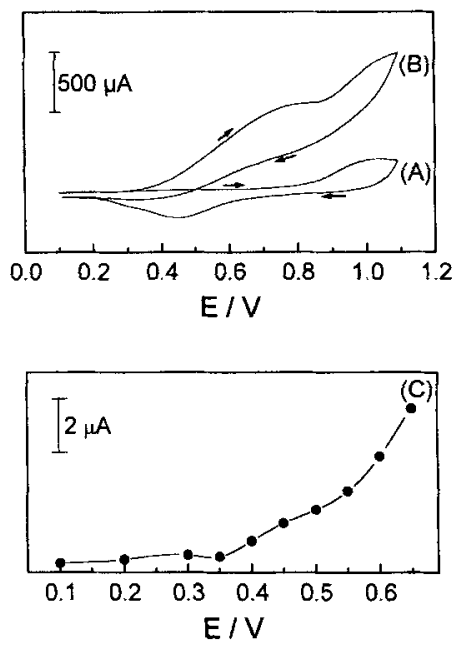

Fig. 4. Current-potential results for hydrogen peroxide oxidation at a thinfilm gold electrode under BIA conditions. Cyclic voltammograms, scan rate $500 \mathrm{mV} \mathrm{s}^{-1}$, flow rate $23 \mu \mathrm{L} / \mathrm{s}$, recorded during injection of: A) $100 \mu \mathrm{L}$ phosphate buffer; B) $5 \mathrm{mM}$ hydrogen peroxide. C) Point-by point hydrodynamic voltammogram based on fixed potential detection, flow rate $64.5 \mu \mathrm{L} / \mathrm{s}$, injections of $50 \mu \mathrm{L}$ of $1 \mathrm{mM}$ hydrogen peroxide.

recorded during the injection of $100 \mu \mathrm{L} 5 \mathrm{mM}$ hydrogen peroxide solution. This shows that the oxidation of hydrogen peroxide starts at $0.35 \mathrm{~V}$ and a current plateau is reached at $0.75 \mathrm{~V}$. In addition, point-by-point hydrodynamic voltammograms were constructed by measuring the BIA response at various fixed detection potentials. Prior to each measurement, the electrode system was equilibrated for about $5 \mathrm{~min}$ at the measuring potential and after recording the amperometric signal, it was stepped directly to the next measuring potential. The representative hydrodynamic voltammogram shown in Figure 4C exhibits a region of decreased slope in the $i-E$ curve between $0.45 \mathrm{~V}$ and $0.55 \mathrm{~V}$. Amperometric detection can therefore be performed applying a working electrode potential of $0.475 \mathrm{~V}$. In contrast to this, Johnston et al. [1] reported that thin-film electrodes produced by deposition of thermally evaporated gold on PVC substrate show no response to hydrogen peroxide up to $0.8 \mathrm{~V}$ (vs. SCE) unless palladium is codeposited. This suggests that the type of deposition procedure and details of preparation conditions significantly affects the voltammetric properties of thin-film gold electrodes.

The major problem with constant potential (DC) amperometric detection of hydrogen peroxide in these experimental configuration is the limited stability of the electrode response, illustrated by the decrease of subsequent BIA signals shown in Figure 5. Similar behavior was observed applying more positive potentials up to $1.00 \mathrm{~V}$. In order to improve the reliability of hydrogen peroxide determinations several activation procedures were tested. Amongst them application of a sequence of pulses gave the most reproducible results which can be characterized as follows. In order to form an activated surface state, application of a potential equal to or higher than $0.7 \mathrm{~V}$ is necessary. Surprisingly, when the measurement is done after a subsequent potential step at potentials lower even than $0.3 \mathrm{~V}$ a well-defined hydrogen peroxide signal is obtained. It is possible to detect hydrogen peroxide if the second (measuring) potential was $0 \mathrm{~V}$ which is the limit for proper electrode function of the thin film electrode in phosphate buffer. This suggests that after applying a potential pulse in the region of formation of the catalytically active oxide layer, detection of hydrogen peroxide can be performed at lower potentials, owing to some of the electrocatalytic activity being retained.

The most reliable results were obtained by applying a triple-pulse program consisting of the following pulse sequence: $0.8 \mathrm{~V}(0.05 \mathrm{~s})$,

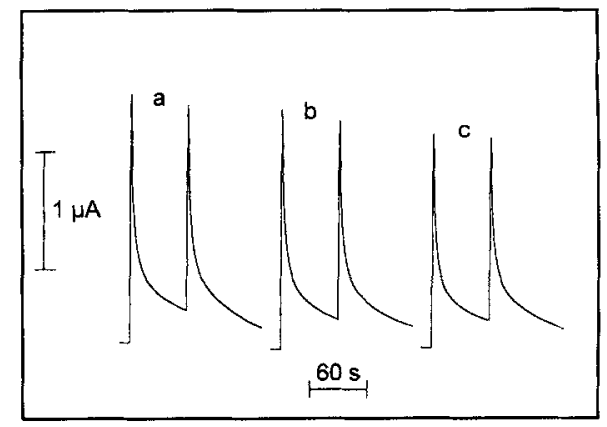

Fig. 5. BIA signals obtained for sequential injections of $50 \mu \mathrm{L}$ of $1 \mathrm{mM}$ hydrogen peroxide onto a thin-film gold electrode. Flow rate $64.5 \mu \mathrm{L} / \mathrm{s}$; $E=+0.475 \mathrm{~V}$; time interval between pairs of injections $5 \mathrm{~min}$.

$0.2 \mathrm{~V}(0.03 \mathrm{~s}), 0.475 \mathrm{~V}(0.05 \mathrm{~s})$. Switching between $0.8 \mathrm{~V}$ and $0.2 \mathrm{~V}$, associated with partial oxidation and reduction of the gold surface, ensures that the electrode characteristics remain constant. Current sampling was done at the actual detection potential of $0.475 \mathrm{~V}$ and each data point is the average of samples taken during $20 \mathrm{~ms}$. The criteria for the choice of the optimized pulse sequence are achievement of stable detector response and a high frequency of data acquisition. The performance characteristics of the optimized multiple pulse detection were studied by performing BIA. Figure 6 demonstrates the good reproducibility of triple-pulse detection for consecutive injections of hydrogen peroxide solution. Repetitive measurements of 20 injections ( $50 \mu \mathrm{L}$ of $1 \mathrm{mM}$ hydrogen peroxide) under identical conditions did not yield any systematic change in the BIA signal and a relative standard deviation of $1.1 \%$ was obtained.

Another interesting feature shown in Figure 6 is that the pulsed detection mode leads to much narrower BIA peaks than obtained with dc-amperometric detection (see Fig. 5 for comparison). Thus it can be concluded that in the case of DC-amperometric detection the current decay is also affected by slow electrode processes of reestablishing the original surface state while with pulsed amperometric detection the mass transport dominates the current decay after reaching the maximum.

Figure 7 shows BIA responses for hydrogen peroxide injections in different concentration ranges. A linear calibration plot was obtained for hydrogen peroxide concentrations between $10^{-4} \mathrm{M}$ and $8 \times 10^{-4} \mathrm{M}$ (regression coefficient: $0.9997, n=5$ ). The limit of detection (calculated for the signal that is twice as much as the baseline noise) is $2 \times 10^{-6} \mathrm{M}$. At concentrations higher than $10^{-3} \mathrm{M}$ the calibration plot is not linear. However, well defined signals could be recorded up to hydrogen peroxide concentrations of $5 \times 10^{-3} \mathrm{M}$ as illustrated in Figure $7 \mathrm{~B}$.

On the basis of the analytical characteristics described above the thin-film gold electrode appears to be a suitable material for future

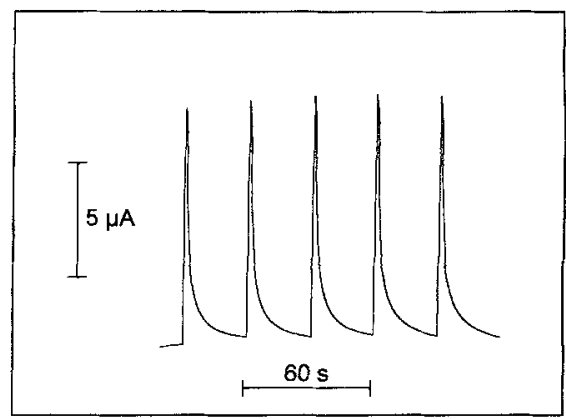

Fig. 6. Reproducibility of BIA at a thin-film gold electrode using triple-pulse amperomentric detection. Injections of $50 \mu \mathrm{L}$ of $1 \mathrm{mM}$ hydrogen peroxide, flow rate $64.5 \mu \mathrm{L} / \mathrm{s}$. 

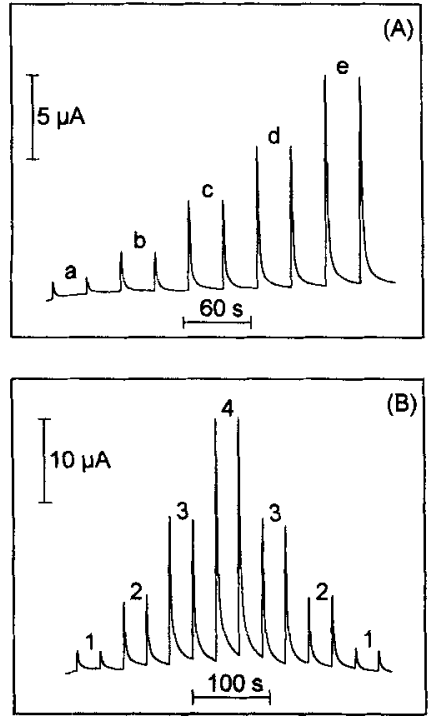

Fig. 7. Determination of hydrogen peroxide by BIA at a thin-film gold electrode using triple-pulse amperometric detection, flow rate $64.5 \mu \mathrm{L} / \mathrm{s}$, $50 \mu \mathrm{L}$ injections of hydrogen peroxide: A: a) $0.1 \mathrm{mM}$, b) $0.2 \mathrm{mM}$, c) $0.4 \mathrm{mM}$, d) $0.6 \mathrm{mM}$, e) $1 \mathrm{mM}$; B: 1) $0.5 \mathrm{mM}$, 2) $1 \mathrm{mM}$, 3) $2 \mathrm{mM}$, 4) $5 \mathrm{mM}$.

developments of enzyme electrodes that involve hydrogen peroxide detection.

\section{Conclusions}

A new type of thin-film gold electrode produced by magnetron sputtering of gold thin films on PVC sheets has been introduced. The voltammetric behavior of this material is comparable to that of conventional polycrystalline gold electrodes exhibiting the typical regions of oxide formation and reduction in neutral and alkaline solutions. However, some restrictions in its use were encountered in acid solutions and in the negative potential range.

The mechanical properties of the plastic foil substrate permit easy construction of electrodes of any shape. This was demonstrated by preparing circular shaped electrodes which could be employed as working electrodes for BIA using a standard voltammetric cell configuration, i.e. introducing all electrodes from the top of the cell.

With respect to future applications of this inexpensive electrode material for disposable enzyme sensors which are based on the detection of hydrogen peroxide, BIA of hydrogen peroxide as analyte was performed. A special multiple-pulse activation and voltammetric detection scheme was developed and shown to give more reliable results than conventional fixed potential amperometry.

\section{Acknowledgement}

This work was supported by a JNICT grant Project PSAU/1532/92.

\section{References}

[1] D.A. Johnston, M.F. Cardosi, D.H. Vaughan, Electroanalysis 1995, 7, 520.

[2] S. Nakao, K. Saitoh, M. Ikeyama, H. Niwa, S. Tanemura, Y. Miyagawa, S. Miyagawa, Surf. Coat. Technol. 1994, 66, 464.

[3] Y. Morishige, S. Kishida, Appl. Phys. A: Solids Surf, 1994, 59, 395.

[4] S.W. Feng, J.J. Nainaparampil, M.F. Tabet, F.K. Urban III, Thin Solid Films $1994,253,402$.

[5] C. Bocking, C. Dineen, Trans. Instrum. Met. Finish. 1994, 72, 101

[6] M. Gilmartin, J.P. Hart, Analyst 1992, 117, 1299.

[7] J.D. Newman, A.P.F. Turner, Anal. Chim. Acta 1992, 262, 13

[8] P.I. Hilditch, M.J. Green, Analyst 1991, 116, 1217.

[9] J. Wang, Analyst 1994, 119, 763.

[10] C.M.A. Brett, A.M. Oliveira Brett, L.C. Mitoseriu, Anal. Chem. 1994, 66, 3145.

[11] D.C. Johnson, W.R. LaCourse, Anal. Chem. 1990, 62, 589 A.

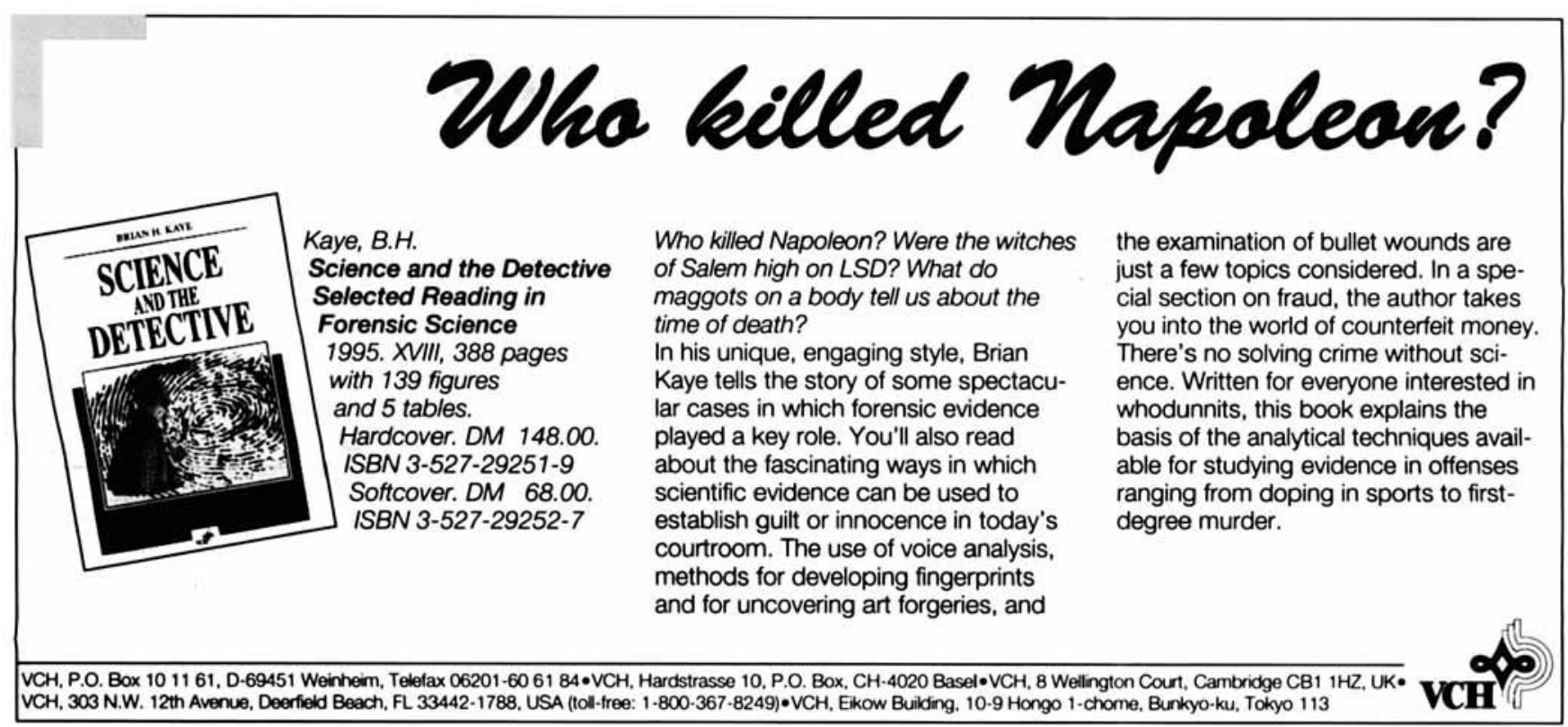

